\title{
A Rank Two Algorithm for Unconstrained Minimization
}

\author{
By Ronald Bass
}

\begin{abstract}
A stable second-order unconstrained minimization algorithm with quadratic termination is given. The algorithm does not require any one-dimensional minimizations. Computational results presented indicate that the performance of this algorithm compares favorably with other well-known unconstrained minimization algorithms.
\end{abstract}

Introduction. Algorithms for unconstrained minimization have enjoyed a great deal of attention in recent years. The fundamental philosophy behind most of these algorithms is the exploitation of the locally quadratic nature of a well-behaved function at an unconstrained local minimum. The Davidon-Fletcher-Powell method [1] was highly successful in this regard and guarantees finite convergence when the function is quadratic, monotone decrease of function value when it is not. However, a potentially time consuming one-dimensional minimization is required in each iteration. Recently developed rank one [2] and rank two [3] methods eliminate the need for this minimization. The rank one method, however, sacrifices finite convergence for stability, and the rank two method eliminates the one-dimensional minimization by trading finite convergence for monotone convergence. The amount of computation in each iteration is reduced, but the number of iterations required may be increased.

In this paper, we present a rank two method which has the combined virtues of finite convergence for quadratic functions and stability for any function, and does not require a one-dimensional search at each iteration. This combination of desirable properties is achieved by making full use of the flexibility of a rank two algorithm.

The algorithm given here is cyclic, i.e., it repeats itself every $N$ iterations (when minimizing a function of $N$ variables) unlike the Davidon-Fletcher-Powell algorithm, which is the same in every iteration. Kelley and Myers [8] presented a cyclic method which is a special case of the algorithm given here.

Statement of the Problem. We are interested in minimizing a scalar function $f(x), x$ an $N$-vector. Let $x^{*}$ be the value of $x$ that minimizes $f$. Assume that $f$ is locally quadratic about $x^{*}$, i.e., for $x$ near $x^{*}$,

$$
f(x)=\frac{1}{2} x^{\prime} G x+b^{\prime} x+e,
$$

where $G$ is a positive definite symmetric matrix (p.d.s.m.). A necessary and sufficient condition that $x^{*}$ minimize this quadratic is that

Received December 21, 1970, revised July 27, 1971.

AMS 1969 subject classifications. Primary 9058.

Key words and phrases. Optimization, unconstrained optimization, unconstrained minimization, conjugate gradient, rank two, nonlinear equation, quasi-newton, ascent, steepest ascent.

Copyright $\odot$ 1972, American Mathematical Society 


$$
g\left(x^{*}\right) \triangleq \operatorname{grad} f\left(x^{*}\right)=0=G x^{*}+b
$$

or

$$
x^{*}=-G^{-1} b .
$$

Given any $x$.

$$
x^{*}-x=-G^{-1} b-x=-G^{-1}(b+G x)=-G^{-1} g(x) .
$$

Thus, if $f$ is locally quadratic about $x^{*}$ and $x$ is near $x^{*}$,

$$
s=-G^{-1} g(x), \quad x^{*}=x+s,
$$

i.e., $s$ is a good direction in which to search for a minimum of $f$. Further, if $x$ is far from $x^{*}$, and $G$ is the matrix of second derivatives of $f$ at $x, s$ is the best direction, based on a local quadratic approximation, in which to search for a decrease in $f$.

It is therefore desirable to have an efficient method for obtaining a good estimate $H$ of the inverse second derivative matrix $G^{-1}$ at $x$.

$N$-Term Rank One Decompositions of p.d.s.m. Let $A$ be an $N \times N$ p.d.s.m. Let $d_{1}, \cdots, d_{N}$ be $A$-orthogonal, i.e.,

$$
\begin{aligned}
d_{i}^{\prime} A d_{i} & =0 & & \text { if } i \neq j, \\
& =r_{i} & & \text { if } i=j, r_{i}>0 ;
\end{aligned}
$$

then it is readily verified that

$$
A^{-1}=\sum_{i=1}^{N} \frac{d_{i} d_{i}^{\prime}}{d_{i}^{\prime} A d_{i}} .
$$

Conversely, if for some (linearly independent) set of vectors, $d_{1}, \cdots, d_{N}$.

$$
A^{-1}=\sum_{i=1}^{N} \frac{1}{r_{i}} d_{i} d_{i}^{\prime},
$$

then, those vectors are $A$-orthogonal and satisfy Eq. (4). Thus, all $N$-term symmetric rank one decompositions of a p.d.s.m. $A^{-1}$ are exactly those decompositions given by all sets of $A^{-1}$-orthogonal vectors.

Constructing a Set of $G$-Orthogonal Vectors From a Set of Linearly Independent Vectors. In the following two lemmas, algorithms are given for constructing $A^{-1}$ orthogonal and $A$-orthogonal vectors for p.d.s.m. $A$ from a set of linearly independent vectors.

Lemma 1. Let $A$ be an $N \times N$ p.d.s.m. Let $d_{1}, \cdots, d_{N}$ be a set of nonzero vectors. Let $A_{0}=0$, and, for $k=1, \cdots, N$, let

$$
\begin{array}{rlrl}
c_{k} & =d_{k}-A_{k-1} A d_{k}, & \\
A_{k} & =A_{k-1}+c_{k} c_{k}^{\prime} / c_{k}^{\prime} A c_{k} & & \text { if } c_{k}^{\prime} A c_{k} \neq 0, \\
& =A_{k-1} & & \text { if } c_{k}^{\prime} A c_{k}=0 .
\end{array}
$$

Then:

(1) There exist $\left\{a_{i j}\right\},\left\{b_{i j}\right\}$ such that 
(a)

$$
\begin{aligned}
c_{i} & =d_{i}+\sum_{i=1}^{i-1} a_{i j} d_{i}, \\
d_{i} & =\sum_{i=1}^{j} b_{i j} c_{i} .
\end{aligned}
$$

(2) If and only if $d_{1}, \cdots, d_{n}, n \leqq N$, are linearly independent, then $c_{1}, \cdots, c_{n}$ are $A$-orthogonal, and therefore if $d_{1}, \cdots, d_{N}$ are linearly independent, $A_{N}=A^{-1}$.

(3) If $d_{1}, \cdots, d_{i-1}$ are linearly independent and $d_{i}$ is linearly dependent on $d_{1}, \cdots$, $d_{i-1}$, then $c_{i}=0$.

(4) $A_{k} A A_{k}=A_{k}$.

Proof. (1a) We have, by definition, $c_{1}=d_{1}$. Assume

$$
c_{i}=\sum_{i=1}^{i} a_{i j} d_{i}, \quad j=1, \cdots, k-1, a_{i j}=1,
$$

where some of the $c_{j}$ may be zero.

Then, by definition of $c_{k}$ and $A_{k}$,

$$
\begin{aligned}
c_{k} & =d_{k}-A_{k-1} A d_{k}=d_{k}-\sum_{j=1 ; c_{j} \sim 0}^{k-1} c_{j} \frac{c_{j}^{\prime} A d_{k}}{c_{j}^{\prime} A c_{j}} \\
& =d_{k}-\sum_{i=1 ; c_{j} \sim 0}^{k-1} \sum_{i=1}^{j} a_{i j} d_{i} \frac{c_{j}^{\prime} A d_{k}}{c_{j}^{\prime} A c_{j}} .
\end{aligned}
$$

(b) follows immediately from (a).

(2) Assume $d_{1}, \cdots, d_{n}$ are linearly independent. We will show that $c_{1}, \cdots, c_{n}$ are $A$-orthogonal. From (1a) of Lemma $1, c_{1}, \cdots, c_{n}$ are nonzero, and

$$
c_{1}^{\prime} A c_{2}=d_{1}^{\prime} A I-\frac{d_{1} d_{1}^{\prime} A}{d_{1}^{\prime} A d_{1}} d_{2}=0 .
$$

Let the inductive hypothesis be that $c_{i}, i \leqq k<n$ are $A$-orthogonal. Then, for $i \leqq k$,

$$
\begin{aligned}
c_{i}^{\prime} A c_{k+1} & =c_{i}^{\prime} A\left(I-A_{k} A\right) d_{k+1} \\
& =\left(c_{i}^{\prime} A-c_{i}^{\prime} A \sum_{i=1}^{k} \frac{c_{j} c_{j}^{\prime} A}{c_{i}^{\prime} A c_{j}}\right) d_{k+1}=0
\end{aligned}
$$

and $c_{1}, \cdots, c_{k+1}$ are $A$-orthogonal.

Conversely, suppose $d_{1}, \cdots, d_{n}$ are linearly dependent. We will show that $c_{1}, \cdots, c_{n}$ are not $A$-orthogonal. From (1a) of Lemma $1, c_{1}, \cdots, c_{n}$ are also linearly dependent, and for some $j \leqq n$,

$$
c_{i}=\sum_{k \neq i} a_{k} c_{k}, \quad a_{l} \neq 0 \text { for some } l \neq j .
$$

Then, if $c_{1}, \cdots, c_{n}$ are $A$-orthogonal,

$$
0=c_{j}^{\prime} A c_{l}=a_{l} c_{l}^{\prime} A c_{l} \neq 0, \text { a contradiction. }
$$

(3) Let $d_{i}=\sum_{i=1}^{i-1} e_{i} d_{i}$.

From (1a) of Lemma 1, we have

$$
c_{i}=d_{i}+\sum_{i=1}^{i-1} a_{i j} d_{i}=\sum_{i=1}^{i-1}\left(a_{i j}+e_{i}\right) \sum_{i=1}^{i} b_{l i} c_{l}=\sum_{i=1}^{i-1} h_{i} c_{i}
$$


and from (1b) of Lemma 1,

$$
d_{i}=\sum_{i=1}^{j} b_{i j} c_{i}=b_{i j} \sum_{i=1}^{i-1} h_{i} c_{i}+\sum_{i=1}^{i-1} b_{i j} c_{i}=\sum_{i=1}^{i-1} m_{i} c_{i} .
$$

Then, by (2) of Lemma 1,

$$
c_{i}=d_{i}-A_{i-1} A d_{i}=\sum_{i=1}^{i-1} m_{i} c_{i}-\sum_{i=1}^{i-1} \frac{c_{i} c_{i}^{\prime}}{c_{i}^{\prime} A c_{i}} A \sum_{i=1}^{i-1} m_{i} c_{i}=0 .
$$

(4) Since, by (2) of Lemma $1, c_{1}, \cdots, c_{k}$ are $A$-orthogonal or zero,

$$
A_{k} A A_{k}=\sum_{i=1 ; c_{i} \neq 0}^{k} \sum_{j=1 ; c_{j} \neq 0}^{k} \frac{c_{i} c_{i}^{\prime}}{c_{i}^{\prime} A c_{i}} A \frac{c_{j} c_{j}^{\prime}}{c_{i}^{\prime} A c_{i}}=A_{k} \text {. }
$$

LEMMA 2. Let $A$ be an $N \times N$ p.d.s.m. Let $d_{1}, \cdots, d_{N}$ be a set of nonzero vectors. Let $A_{1}=A$ and, for $k=1, \cdots, N$, let

$$
\begin{aligned}
c_{k} & =A_{k} d_{k}, & & \\
A_{k+1} & =A_{k}-c_{k} c_{k}^{\prime} / c_{k}^{\prime} d_{k} & & \text { if } c_{k}^{\prime} d_{k} \neq 0, \\
& =A_{k} & & \text { if } c_{k}^{\prime} d_{k}=0 .
\end{aligned}
$$

Then:

(1) Let $z^{\prime} A d_{i}=0, i=1, \cdots, k-1$. Then $A z=A_{k} z$.

(2) $A_{k}$ is positive semidefinite and $A_{k} d_{i}=0, j<k$.

(3) Let $j$ be the number of linearly independent vectors in $d_{1}, \cdots, d_{k-1}$. Then $\operatorname{rank}\left(A_{k}\right)=N-j$.

Further, if and only if $d_{1}, \cdots, d_{N}$ are linearly independent,

(4) $A_{N+1}=0$, i.e. $A=\sum_{i=1}^{N} c_{i} c_{i}^{\prime} / c_{i}^{\prime} d_{i}$.

(5) $c_{1}, \cdots, c_{N}$ are $A^{-1}$-orthogonal.

Proof. (1) Choose $z \neq 0$ such that $z^{\prime} A d_{1}=0$. Then,

$$
A_{2} z=A z-\frac{A d_{1} d_{1}^{\prime} A z}{d_{1}^{\prime} A d_{1}}=A z \text {. }
$$

Let the inductive hypothesis be that $A_{k} z=A z$ for all $z$ such that $z^{\prime} A d_{i}=0, j=1, \cdots$, $k-1$. Then, certainly, $A_{k} z=A z$ for all $z$ such that $z^{\prime} A d_{i}=0, j=1, \cdots, k$, and, for all such $z$,

$$
\begin{aligned}
A_{k+1} z & =A_{k} z-\frac{A_{k} d_{k} d_{k}^{\prime} A_{k}}{d_{k}^{\prime} A_{k} d_{k}} z=A_{k} z=A z, & & \text { if } c_{k}^{\prime} d_{k} \neq 0, \\
& =A_{k} z=A z, & & \text { if } c_{k}^{\prime} d_{k}=0 .
\end{aligned}
$$

(2) By definition, $A_{1}$ is positive definite. For $d_{1} \neq 0$,

$$
A_{2} d_{1}=\left(A-\frac{A d_{1} d_{1}^{\prime} A}{d_{1}^{\prime} A d_{1}}\right) d_{1}=0 .
$$

Let the inductive hypothesis be that $A_{k}$ is positive semidefinite and $A_{k} d_{i}=0, j<k$. We will show that $A_{k+1}$ is positive semidefinite and $A_{k+1} d_{i}=0, j<k+1$.

Clearly, any $x$ may be written

$$
x=z+\sum_{i=1}^{k} a_{i} d_{i}, \quad z^{\prime} A d_{i}=0, \quad i \leqq k .
$$


Then, $z^{\prime} A=z^{\prime} A_{k}$ by (1) of Lemma 2 , so that $z^{\prime} A_{k} d_{i}=0, i \leqq k$, and since by hypothesis $A_{k} d_{i}=0, j<k$, it is easily seen that

$$
x^{\prime} A_{k+1} x=z^{\prime} A_{k} z \geqq 0 .
$$

Thus, $A_{k+1}$ is positive semidefinite. By definition, $A_{k+1} d_{j}=0, j<k$. If $d_{k}$ is linearly dependent on $d_{1}, \cdots, d_{k-1}$, then $A_{k} d_{k}=0$ and $A_{k+1}=A_{k}$. Otherwise, $d_{k}^{\prime} A_{k} d_{k}>0$, since $A_{k}$ is positive semidefinite and, by definition, $\operatorname{rank}\left(A_{k}\right)$ is at least $N-(k-1)$. Then,

$$
A_{k+1} d_{k}=A_{k} d_{k}-A_{k} d_{k} \frac{d_{k}^{\prime} A_{k} d_{k}}{d_{k}^{\prime} A_{k} d_{k}}=0 .
$$

This completes the induction.

(3) By definition, $\operatorname{rank}\left(A_{k}\right) \geqq N-j$, and by (2) of Lemma $2, \operatorname{rank}\left(A_{k}\right) \leqq N-j$.

(4) By (3) of Lemma 2, rank $A_{N+1}=0$ if and only if $d_{1}, \cdots, d_{N}$ are linearly independent.

(5) We need only note that $c_{k}^{\prime} d_{k}=d_{k}^{\prime} A_{k} d_{k}>0$; then, by (4) of Lemma 2 and Eq. (5b), $c_{k}^{\prime} d_{k}=c_{k}^{\prime} A^{-1} c_{k}$ and $c_{1}, \cdots, c_{N}$ are $A^{-1}$-orthogonal.

Let $f$ be given by Eq. (1). Now, suppose we have an algorithm for minimizing $f$ such that in the $k$ th iteration we take a step $d_{k}=x_{k+1}-x_{k}$, resulting in a gradient change $y_{k}=g_{k+1}-g_{k}$.

We show in Lemma 3 how to construct $N G$-orthogonal vectors from any $N$ steps (such that $d_{1}, \cdots, d_{N}$ are linearly independent) without using $G$ or $G^{-1}$ explicitly.

Lemma 3. Let $d_{1}, \cdots, d_{N}$ be linearly independent. Let $H_{0}=0$. Let $H_{k}=H_{k-1}+$ $s_{k} s_{k}^{\prime} / s_{k}^{\prime} y_{k}$, where $s_{k}=d_{k}-H_{k-1} y_{k}$. Then, $H_{N}=G^{-1}$.

Proof. We will show that

$$
s_{k}^{\prime} G s_{k}=d_{k}^{\prime} y_{k}-y_{k}^{\prime} H_{k-1} y_{k}=s_{k}^{\prime} y_{k} .
$$

Then, it follows from Lemma 1 and the fact that

$$
s_{k}=d_{k}-H_{k-1} y_{k}=d_{k}-H_{k-1} G d_{k}
$$

that $s_{1}, \cdots, s_{N}$ are $G$-orthogonal and $G^{-1}=H_{N}$. Clearly,

$$
s_{1}=d_{1} \text { and } s_{1}^{\prime} y_{1}=d_{1}^{\prime} y_{1}=d_{1}^{\prime} G d_{1}=s_{1}^{\prime} G s_{1} .
$$

Then, by Lemma $1, H_{1} G H_{1}=H_{1}$. Let the inductive hypothesis be that

$$
H_{k-1} G H_{k-1}=H_{k-1} \text {. }
$$

Then, since $G d_{k}=y_{k}$, we have

$$
\begin{aligned}
s_{k}^{\prime} G s_{k} & =\left(d_{k}-H_{k-1} y_{k}\right)^{\prime} G\left(d_{k}-H_{k-1} y_{k}\right) \\
& =d_{k}^{\prime} G d_{k}-y_{k}^{\prime} H_{k-1} G d_{k}-d_{k}^{\prime} G H_{k-1} y_{k}+y_{k}^{\prime} H_{k-1} G H_{k-1} y_{k} \\
& =d_{k}^{\prime} y_{k}-y_{k}^{\prime} H_{k-1} y_{k}=s_{k}^{\prime} y_{k},
\end{aligned}
$$

and, by (4) of Lemma 1 ,

$$
H_{k} G H_{k}=H_{k} .
$$

Note that the formula for updating $H_{k}$ in Lemma 3 is the same formula used in the rank one algorithm (Eq. (6)). However, in Lemma 3, $H_{0}=0$, whereas in the rank one algorithm $H_{0}$ is arbitrary. 
It should be noted that Lemmas 1 and 2 are simply applications of the GramSchmidt orthogonalization procedure with respect to the inner product defined by $A$ or $A^{-1}$. The matrices $A_{k}$ in these lemmas are $A$-orthogonal and $A^{-1}$-orthogonal projection operators. Lemma 3 is an application of Lemma 1.

Desirable Properties for $H_{k}$. Suppose $f$ is given by Eq. (1), and let $d_{k}=x_{k+1}$ $x_{k}, y_{k}=g_{k+1}-g_{k}, G^{-1}=H$. Then,

$$
H y_{k}=G^{-1}\left(G x_{k+1}+b-G x_{k}-b\right)=d_{k} .
$$

We can now give two definitions of a "good" estimate $H_{k}$ of $G^{-1}$.

First, we could require that $H_{k+1}-H_{k}$ be of rank one and that

$$
H_{k+1} y_{k}=d_{k} \text {, }
$$

in which case

$$
H_{k+1}=H_{k}+\frac{\left(d_{k}-H_{k} y_{k}\right)\left(d_{k}-H_{k} y_{k}\right)^{\prime}}{\left(d_{k}-H_{k} y_{k}\right)^{\prime} y_{k}}
$$

is the only possible such formula for updating $H_{k}$ [2]. Alternately, we can require that

$$
H_{k}=\sum_{i=1}^{k} \frac{c_{i} c_{i}^{\prime}}{c_{i}^{\prime} G c_{i}}+\sum_{i=k+1}^{N} z_{i} z_{i}^{\prime}
$$

where $\left(c_{1}, \cdots, c_{k}, z_{k+1}, \cdots, z_{N}\right)$ is a set of linearly independent vectors and $c_{1}, \cdots, c_{k}$ are $G$-orthogonal vectors constructed from the first $k$ steps as described in Lemma 3. This criterion motivates the algorithm given below.

We have shown that a good search direction, $-H_{k} g_{k}$, can be obtained if $H_{k}$ is a "good" estimate of the inverse second derivative matrix of $f$ (we will use the second definition given above, i.e., Lemma 3). Thus, $H_{k}$ should be updated so that if $f$ is quadratic, $H_{N}=G^{-1}$ (quadratic termination property). This property ensures rapid convergence after reaching a point $x$ near $x^{*}$ if $f$ is quadratic locally about $x^{*}$. Further, it is desirable that $H_{k}$ be positive definite. Then, no matter how poor an estimate is $H_{k}$ of the inverse second derivative matrix, the "best" search direction, $-H_{k} g_{k}$, is a locally downhill direction, i.e., there is some positive $t$ such that $f\left(x_{k}+t\left(-H_{k} g_{k}\right)\right)$ $<f\left(x_{k}\right)$ (stability property). In the algorithm given below, $H_{k}$ has both of these properties.

Algorithm for Unconstrained Minimization. We now give an outline of an unconstrained minimization algorithm and show in detail how $H_{k}$ is updated. Choice of step direction and step size are discussed later.

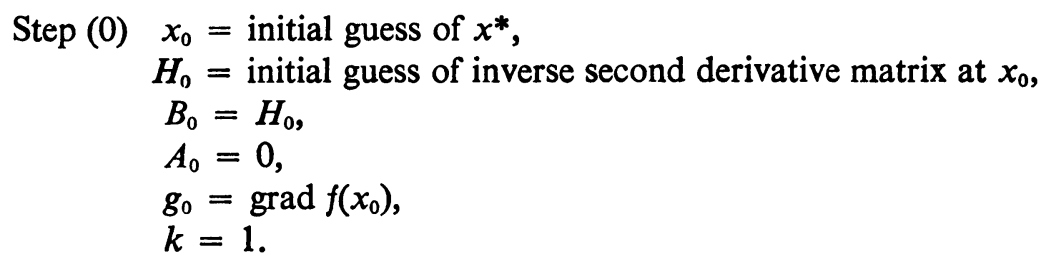

Step (1) (choose a step $d_{k}$ ),

$x_{k}=x_{k-1}+d_{k}$,

(compute $f\left(x_{k}\right), g_{k}$ and test for convergence), 


$$
\begin{aligned}
& y_{k}=g_{k}-g_{k-1}, \\
& s_{k}=d_{k}-A_{k-1} y_{k}, \\
& a_{k}=s_{k}^{\prime} y_{k}, \\
& \text { if } a_{k} \leqq 0 \text { go to Step (3) (see discussion below). }
\end{aligned}
$$

Step (2) $A_{k}=A_{k-1}+s_{k} s_{k}^{\prime} / a_{k}$

$B_{k}=B_{k-1}-B_{k-1} S_{k} s_{k}^{\prime} B_{k-1} / s_{k}^{\prime} B_{k-1} s_{k}$,

$H_{k}=A_{k}+B_{k}$,

increase $k$ by 1 ,

if $k \leqq N$ go to Step (1),

if $k>N$ go to Step (3).

Step (3) set $H_{0}=B_{0}=H_{k}$,

$$
\begin{aligned}
& A_{0}=0, \\
& x_{0}=x_{k}, \\
& k=1, \\
& \text { go to Step (1). }
\end{aligned}
$$

Properties of the Algorithm. We now exhibit some important properties of the above algorithm.

Let $C_{1}$ be the condition: $d_{1}, \cdots, d_{N}$ are linearly independent.

Property (1) Assume that $a_{1}, \cdots, a_{N}$ are positive. Then, $s_{1}, \cdots, s_{N}$ are linearly independent if and only if $C_{1}$ holds.

Proof. It is sufficient to show that $s_{k}$ is a linear combination of $d_{1}, \cdots, d_{k}$, where the coefficient of $d_{k}$ is not zero. Clearly, $s_{1}=d_{1}$. Let the inductive hypothesis be that

$$
s_{i}=\sum_{i=1}^{i} a_{i j} d_{i}, \quad j=1, \cdots, k-1 .
$$

Then

$$
\begin{aligned}
s_{k} & =d_{k}-A_{k-1} y_{k}=d_{k}-\sum_{i=1}^{k-1} s_{j} \frac{s_{i}^{\prime} y_{k}}{a_{i}} \\
& =d_{k}-\sum_{j=1}^{k-1} \sum_{i=1}^{j} a_{i j} d_{i} \frac{s_{i}^{\prime} y_{k}}{a_{j}} .
\end{aligned}
$$

Property (2) $B_{N}=0$ if and only if $C_{1}$ holds (by (4) of Lemma 2 and Property (1)).

Property (3) Let $f$ be quadratic, $f$ given by Eq. (1). Then

(a) $a_{i}>0$ if and only if $C_{1}$ holds.

Proof. (a) If $f$ is quadratic, then, from the proof of Lemma $3, a_{i}=s_{i}^{\prime} G s_{i}$, and $a_{i}$ is zero if and only if $s_{i}$ is zero, and is positive otherwise. But by (1) and (3) of Lemma 1 and by Property (1), $s_{i}$ is zero if and only if $C_{1}$ does not hold. (b) $H_{N}=G^{-1}$ if and only if $C_{1}$ holds (by Lemma 3 and Property (2)).

Property (4) Let $a_{k}>0$ for all $k$. Then, $H_{k}$ is positive definite for all $k$ if and only if $C_{1}$ holds.

Proof. Assume $C_{1}$ holds. Let $w_{1}, \cdots, w_{k}$ satisfy

$$
\begin{aligned}
& w_{i}=\sum_{j=1}^{k} c_{i i} s_{i}, \quad w_{i}^{\prime} s_{i}=1, \quad i=j, \\
& =0, \quad i \neq j .
\end{aligned}
$$


Then, any vector $x$ can be written as $x=v+y$, where

$$
y=\sum_{i=1}^{k} b_{i} w_{i}, \quad v^{\prime} s_{i}=0, \quad i=1, \cdots, k .
$$

From Lemma 2, $B_{k} s_{i}=0, i=1, \cdots, k$, and $B_{k}$ is of rank $N-k$ and is positive semidefinite. If $y$ and $v$ are not zero, then

$$
\begin{aligned}
y^{\prime} B_{k} y=0, & v^{\prime} B_{k} v>0, \\
y^{\prime} A_{k} y>0, & v^{\prime} A_{k} v=0 .
\end{aligned}
$$

For $x \neq 0, y$ and $v$ are not both zero, and

$$
x^{\prime} H_{k} x=y^{\prime} A_{k} y+v^{\prime} B_{k} v>0 .
$$

Conversely, if $C_{1}$ does not hold, then, by Property (1), for some $k, s_{k}$ is a linear combination of $s_{1}, \cdots, s_{k-1}$, and from (2) of Lemma $2, B_{k-1} s_{k}=0$ and $B_{k}$ is undefined.

Property (5) $H_{k}$ satisfies the second criterion for a good estimate of the inverse second derivative matrix, since $A_{k}$ is in fact constructed as indicated in Lemma 3. As with the other properties, this condition is contingent upon $C_{1}$ being satisfied.

Choice of Step Direction. The natural choice of step direction, as discussed in the introduction, is

$$
d_{k} /\left\|d_{k}\right\|=-H_{k} g_{k} /\left\|H_{k} g_{k}\right\|,
$$

where $\|x\|=\left(x^{\prime} x\right)^{1 / 2}$. However, it is clear from Properties (1)-(5) that if the algorithm is to be well behaved, $C_{1}$ must be satisfied. Suppose $C_{1}$ is violated by $-H_{k} g_{k}$. Then, a satisfactory direction is

$$
\frac{d_{k}}{\left\|d_{k}\right\|}=-\frac{H_{k} g_{k}}{\left\|H_{k} g_{k}\right\|}\left(1-\alpha_{k}^{2}\right)^{1 / 2} \pm \alpha_{k} e_{k},
$$

where $e_{k}$ is any unit vector perpendicular to $d_{1}, \cdots, d_{k-1}$ and $\alpha_{k}$ is some prespecified constant, $0<\alpha_{k}<1$ so that $d_{k} /\left\|d_{k}\right\|$ makes an angle of $\tan ^{-1}\left(1-\alpha_{k}^{2}\right)^{1 / 2} / \alpha_{k}$ with the manifold generated by $d_{1}, \cdots, d_{k-1}$.

The choice of sign is such that $-g_{k}^{\prime} d_{k}$ is maximized, i.e., the modified $d_{k}$ is in the most downhill direction. In practice, an $e_{k}$ component would be added if $-H_{k} g_{k}$ almost violated $C_{1}$, and we would then choose

$$
\frac{d_{k}}{\left\|d_{k}\right\|}=-\frac{H_{k} g_{k}-e_{k}^{\prime} H_{k} g_{k} e_{k}}{\left\|H_{k} g_{k}-e_{k}^{\prime} H_{k} g_{k} e_{k}\right\|}\left(1-\alpha_{k}^{2}\right)^{1 / 2} \pm \alpha_{k} e_{k},
$$

where the sign is again chosen to maximize $-g_{k}^{\prime} d_{k}$ (see Appendix I for an efficient way to compute $e_{k}$ and check for linear independence).

The choice of $-H_{k} g_{k}$ as a search direction is predicated upon the assumption that $H_{k}$ is in some sense a fair approximation of the inverse second derivative matrix. This assumption is not necessarily valid for the first few iterations, and a more rapid initial reduction of the cost function might be obtained by taking these steps in the $-g_{k}$ direction.

Choice of Step Size. In practical application of the Davidon-Fletcher-Powell algorithm, it is not possible to find the exact minimum along the search direction, 
and any method which is used to get an accurate approximation to that minimum, such as a Fibonacci search, is very time consuming. Consequently, some method which gives an approximation to the minimum is usually used, and it is hoped that the inaccuracy will not materially upset the performance of the algorithm. In the algorithm given here, any step size which decreases $f$ is acceptable, and will yield convergence of $H_{k}$ to $G^{-1}$ in $N$ steps if $f$ is quadratic. However, use of a cubic interpolation scheme to achieve approximately the minimum along the search direction, such as that given by Fletcher and Powell will guarantee that if $H_{k}=G^{-1}$, the minimum will be achieved on the $k$ th step, and will also ensure that $g_{k+1}$ is approximately perpendicular to $d_{k}$, which in practice is often sufficient to guarantee that $C_{1}$ is satisfied.

A somewhat more satisfactory method is the following: take a step $d_{k}=-H_{k} g_{k}$; if $f\left(x_{k}+d_{k}\right)<f\left(x_{k}\right)$, let $x_{k+1}=x_{k}+d_{k}$; otherwise, try $x_{k+1}=x_{k}+d_{k} / h^{m}, m=$ $1,2, \cdots$, until a decrease in $f$ is obtained, where, for example, $h=10$ may be used so that $m$ will remain small. This procedure guarantees that if $H_{k}=G^{-1}$, the minimum will be achieved on the $k$ th step, and keeps the number of function evaluation per iteration small by using a relatively large $h$. Of course, if enough function values have been computed $(m=2)$, a cubic interpolation can be used rather than simply using a larger $m$. This combined approach seems to be quite effective in practice (see test problems).

Case when $a_{k} \leqq 0$. Let $G\left(x_{k}\right)$ denote the second derivative matrix at $x_{k}$. We have shown (Property (3)) that if $f$ is quadratic, $a_{k}>0$. If $a_{k} \leqq 0$, then, either $H_{k}$ is not a good estimate of $G^{-1}\left(x_{k}\right)$ or the step size is so large that a quadratic approximation to $f$ with metric $G\left(x_{k}\right)$ is not a good representation of $f$ on the set $\left\{x_{k}+\beta\left(x_{k+1}-x_{k}\right), 0 \leqq \beta \leqq 1\right\}$. In either case, the curvature information in $H_{k}$ is no longer very accurate. Thus, it is desirable to deemphasize the information in $H_{k}$ by treating $H_{k}$ and $x_{k}$ as an initial guess and starting again, rather than assuming that $H_{k}$ is composed of $G$-orthogonal vectors constructed from the last $k$ steps. Therefore, if $a_{k} \leqq 0$ (or $a_{k} \leqq \epsilon, \epsilon>0$ ), we go to Step (3). Then, for the $k$ th step

$$
\begin{aligned}
& s_{k}=s_{1}=d_{k}, \\
& a_{k}=a_{1}=d_{k}^{\prime} y_{k}=d_{k}^{\prime} G d_{k} \quad \text { if } f \text { is quadratic. }
\end{aligned}
$$

If $a_{k}$ is still negative, then $f$ is probably not well approximated by a quadratic on $\left\{x_{k}+\beta d_{k}, 0 \leqq \beta \leqq 1\right\}$; again we let $x_{k+1}=x_{k}+d_{k}$ and return to Step (3) (without updating $A_{0}$ or $B_{0}$ ).

The effect of this procedure is to remove old information from $A_{k}$ while retaining that information in $B_{k}$. The search direction, $-H_{k} g_{k}$, is affected by the old information, but $s_{k}$ depends only on new information in $A_{k}$.

Test Problems. The Davidon-Fletcher-Powell algorithm is apparently the most successful unconstrained minimization algorithm to date, so the examples presented here are taken from Fletcher and Powell's paper [1] and compared with their results. The currently accepted basis for comparison of unconstrained minimization algorithms is the number of objective function evaluations required for convergence, since in most practical problems these consume the bulk of the computing time. Calculation of the gradient is counted as $N$ function evaluations ( $N$ the number of variables), since evaluation of each of the $N$ components of the gradient analytically 
is roughly equivalent to evaluation of the objective function, and evaluation of the gradient by perturbation requires at least $N$ function evaluations at perturbed values of the variables.

Since Fletcher and Powell's algorithm, as applied to their test problems [1], requires at least two function and gradient evaluations per iteration (one for the main algorithm and one for the cubic interpolation), we shall ascribe to their examples a minimum of $2 N+2$ function evaluations per iteration.

The calculations for the algorithm given here were carried out on a Univac 1108 computer in single-precision arithmetic (8 significant figures). In all examples, the step direction and step size schemes given above were used, with $h=10$ and $\alpha_{k}=.1$ and were found to give good results (convergence rates were not very sensitive to changes in these parameters). The first test problem is (Table 1) the parabolic valley

TABLE 1

Parabolic Valley

Our Method

$\begin{array}{ccccc}\text { Iteration } & f & \begin{array}{c}\text { Number of function } \\ \text { evaluations }\end{array} & f & \begin{array}{c}\text { Minimum number of } \\ \text { function evaluations }\end{array} \\ 0 & 24.2 & 1 & 24.2 & 1 \\ 3 & 4.18 & 12 & 3.69 & 18 \\ 6 & 3.67 & 21 & 1.605 & 36 \\ 9 & 3.62 & 30 & .745 & 54 \\ 12 & 3.37 & 40 & .196 & 72 \\ 15 & 2.14 & 50 & .012 & 90 \\ 18 & 1.78 & 60 & 1 \times 10^{-8} & 108 \\ 25 & .631 & 82 & & \\ 30 & .344 & 100 & & \\ 35 & .260 & 116 & & \\ 40 & .116 & 133 & & \\ 45 & .066 & 151 & & \\ 50 & .025 & 17 & & \\ 55 & .015 & 191 & & \\ 58 & .0012 & 203 & & \\ 60 & 2.7 \times 10^{-4} & 210 & & \\ 62 & 3.5 \times 10^{-5} & 217 & & \\ 64 & 1.4 \times 10^{-7} & 224 & & \\ 65 & 4.4 \times 10^{-8} & 228 & & \\ 66 & 4.6 \times 10^{-12} & 231 & & \\ & & & & \end{array}$

originally given by Rosenbrock [5],

$$
f\left(x_{1}, x_{2}\right)=100\left(x_{2}-x_{1}^{2}\right)^{2}+\left(1-x_{1}\right)^{2}
$$

with starting point $(-1.2,1)$ and a zero at $(1,1)$. The second problem (Table 2$)$ is a steep-sided helical valley, 


$$
f\left(x_{1}, x_{2}, x_{3}\right)=100\left[\left(x_{3}-10 A\right)^{2}+(R-1)^{2}\right]+x_{3}^{2},
$$

where

and

$$
\begin{aligned}
2 \pi A & =\arctan \left(x_{2} / x_{1}\right) & & \text { if } x_{1}>0, \\
& =\arctan \left(x_{2} / x_{1}\right)+\pi & & \text { if } x_{1}<0,
\end{aligned}
$$

$$
R=\left(x_{1}^{2}+x_{2}^{2}\right)^{1 / 2} .
$$

\begin{tabular}{|c|c|c|c|c|}
\hline \multirow[b]{3}{*}{ Iteration } & \multicolumn{4}{|c|}{$\begin{array}{c}\text { TABLE } 2 \\
\text { Helical Valley }\end{array}$} \\
\hline & \multicolumn{2}{|c|}{ Our Method } & \multicolumn{2}{|c|}{ Fletcher and Powell } \\
\hline & $f$ & $\begin{array}{c}\text { Number of function } \\
\text { evaluations }\end{array}$ & $f$ & $\begin{array}{l}\text { Minimum number of } \\
\text { function evaluations }\end{array}$ \\
\hline 0 & 2500 & 1 & 2500 & 1 \\
\hline 1 & 2139 & 4 & 520 & 8 \\
\hline 2 & 13.34 & 8 & 110 & 16 \\
\hline 3 & 6.99 & 13 & 74.1 & 24 \\
\hline 4 & 6.84 & 17 & 24.2 & 32 \\
\hline 5 & .46 & 21 & 10.9 & 40 \\
\hline 6 & .14 & 25 & 9.8 & 48 \\
\hline 7 & .065 & 29 & 6.3 & 56 \\
\hline 8 & .049 & 33 & 6.09 & 64 \\
\hline 9 & .047 & 37 & 1.89 & 72 \\
\hline 10 & .047 & 41 & 1.75 & 80 \\
\hline 11 & .040 & 46 & .76 & 88 \\
\hline 12 & .0089 & 50 & .38 & 96 \\
\hline 13 & .0063 & 54 & .14 & 104 \\
\hline 14 & .0045 & 59 & .058 & 112 \\
\hline 15 & $5.6 \times 10^{-4}$ & 63 & .018 & 120 \\
\hline 16 & $1.4 \times 10^{-4}$ & 67 & $8 \times 10^{-4}$ & 128 \\
\hline 17 & $1.1 \times 10^{-4}$ & 72 & $3 \times 10^{-6}$ & 136 \\
\hline 18 & $5.0 \times 10^{-6}$ & 76 & $7 \times 10^{-8}$ & 144 \\
\hline 19 & $3.6 \times 10^{-6}$ & 81 & & \\
\hline 20 & $2.5 \times 10^{-6}$ & 86 & & \\
\hline 21 & $3.7 \times 10^{-9}$ & 90 & & \\
\hline
\end{tabular}

The distance $x_{3}$ along the axis of the helix is restricted so that $-2.5<x_{3}<7.5$. The starting point is $(-1,0,0)$ and the function has a zero minimum at $(1,0,0)$.

Finally, we give some examples (Table 3) of solutions of functions of many variables. The function used is

$$
f(x)=\sum_{i=1}^{N} x_{i}^{2}+\left(\sum_{i=1}^{N} i^{1 / 2} x_{i}\right)^{2}+\left(\sum_{i=1}^{N} i^{1 / 2} x_{i}\right)^{4}
$$

which has a zero minimum at $x_{i}=0$. Starting values were $x_{i}=.1$. 
TABLE 3

Function of Many Variables

\begin{tabular}{|c|c|c|c|c|c|}
\hline \multicolumn{3}{|c|}{$N=10$} & \multicolumn{3}{|c|}{$N=20$} \\
\hline Iteration & $\begin{array}{l}\text { Number of } \\
\text { function } \\
\text { evaluations }\end{array}$ & $\begin{array}{c}\text { Function } \\
\text { value }\end{array}$ & Iteration & $\begin{array}{l}\text { Number of } \\
\text { function } \\
\text { evaluations }\end{array}$ & Function value \\
\hline 0 & 1 & 30.6 & 0 & 1 & 1484 \\
\hline 3 & 37 & .154 & 3 & 69 & 39.1 \\
\hline 6 & 70 & .0035 & 6 & 132 & 1.86 \\
\hline 9 & 103 & .098 & 10 & 176 & .47 \\
\hline 12 & 125 & .00019 & 20 & 386 & .010 \\
\hline 15 & 158 & $6 \times 10^{-7}$ & 30 & 596 & .0017 \\
\hline 18 & 192 & $6 \times 10^{-8}$ & 40 & 806 & .0016 \\
\hline 20 & 205 & $4 \times 10^{-10}$ & 50 & 1017 & $6.6 \times 10^{-4}$ \\
\hline & & & 60 & 1227 & $3.8 \times 10^{-4}$ \\
\hline & & & 70 & 1437 & $2.2 \times 10^{-4}$ \\
\hline & & & 80 & 1649 & $1.7 \times 10^{-4}$ \\
\hline & & & 90 & 1859 & $7.2 \times 10^{-5}$ \\
\hline & & & 100 & 2069 & $6.2 \times 10^{-5}$ \\
\hline & & & 110 & 2279 & $4.9 \times 10^{-5}$ \\
\hline & & & 120 & 2490 & $1.3 \times 10^{-5}$ \\
\hline & & & 125 & 2600 & $1.4 \times 10^{-6}$ \\
\hline & & & 126 & 2621 & $3.1 \times 10^{-7}$ \\
\hline & & & 127 & 2642 & $8.7 \times 10^{-10}$ \\
\hline
\end{tabular}

Conclusions. The above test problems indicate that our results compare favorably with those given by Fletcher and Powell in [1], and that our algorithm is applicable to problems of moderate size. The advantages of this algorithm are that stable finite convergence is obtained in the case of a quadratic objective function without the need for a line search, and there is almost complete freedom in the choice of step direction and step size (the choices used here are certainly not definitive). The main disadvantage of this algorithm seems to be that the need to separately store the $A_{k}$ and $B_{k}$ matrices and to check for linear independence (see Appendix I) requires storage of two more $N \times N$ symmetric matrices than in Fletcher and Powell's algorithm.

It should be noted that in the Davidon-Fletcher-Powell type of algorithm, completely analogous $A_{k}$ and $B_{k}$ matrices are constructed [6]; however, the step directions are chosen to be $G$-orthogonal vectors, and the step direction and size is completely determined by this consideration. Such restrictions are avoided in our algorithm by computing $G$-orthogonal vectors from an almost arbitrary step, rather than requiring the step itself to be $G$-orthogonal.

Finally, we note that minimization of a positive semidefinite quadratic function is treated in Appendix II. We show that with almost no modification, all the above results hold if $A^{-1}$ and $G^{-1}$ are replaced by $A^{*}$ and $G^{*}$ (pseudo-inverse of $A$ and $G$ ) and that the above algorithm may be applied with only slight modification, and 
therefore may be used to minimize functions that have singular second derivative matrices at a minimum.

Appendix I (Gram-Schmidt Orthogonalization Procedure). Let $d_{1}, \cdots, d_{k}$ be step directions. Let

$$
\begin{aligned}
& P_{0}=0, \quad c_{k}=d_{k}-P_{k-1} d_{k}, \\
& P_{k}=P_{k-1}+c_{k} c_{k}^{\prime} / c_{k}^{\prime} c_{k} \text { if } c_{k} \neq 0, \\
&=P_{k-1} \text { if } c_{k}=0 .
\end{aligned}
$$

Then $P_{k-1} d_{k}$ is the projection of $d_{k}$ on the manifold generated by $d_{1}, \cdots, d_{k-1}$. The angle between this manifold and $d_{k}$ is $\cos ^{-1}\left(\left\|P_{k-1} d_{k}\right\| /\left\|d_{k}\right\|\right)$. Thus, $d_{k}$ is "almost" linearly dependent on $d_{1}, \cdots, d_{k-1}$, if $\left|1-\left\|P_{k-1} d_{k}\right\| /\left\|d_{k} \mid\right\|\right.$ is "almost" zero.

Clearly, the columns of $M, M=I-P_{k-1}$, where $I$ is the identity, are orthogonal to $d_{1}, \cdots, d_{k-1}$. Let $m_{i}$ be the $j$ th column of $M, j$ chosen so that $m_{i} \neq 0$. Let $u_{i}$ be a vector with the $j$ th component 1 and all other components zero. Let $p_{i}$ be the $j$ th column of $P_{k-1}$. Then a unit vector $e_{k}$ orthogonal to $d_{1}, \cdots, d_{k}$ is

$$
e_{k}=\frac{m_{i}}{\left\|m_{i}\right\|}=\frac{u_{i}-P_{i}}{\left\|u_{i}-p_{j}\right\|}
$$
Let

Appendix II (Minimization of a Positive Semidefinite Quadratic Function).

$$
f(x)=\frac{1}{2} x^{\prime} A x+b^{\prime} x+e,
$$

where $A$ is a positive semidefinite symmetric matrix (p.s.s.m.). The corollary to Lemma 1 given below may be used to modify the algorithm to include the case where $A$ is a p.s.s.m.

Notation.

$N_{A}=$ null space of $A$,

$P_{A}=N_{A}^{\perp}$ (orthogonal complement of $N_{A}$ ),

$R_{A}=$ range of $A$.

Definition. $A^{*}$ is the pseudo-inverse of $A$ if $A^{*}$ satisfies

$$
\begin{array}{rlrl}
A A^{*} x & =x, & & x \text { in } P_{A}, \\
A^{*} x & =0, & x \text { in } R_{A}^{\perp},
\end{array}
$$

(for definition and properties of $A^{*}$, see [7, Appendix C]). We note that $\left(A^{*}\right)^{*}=A$, and $A A^{*} A=A$. Further, we have from the symmetry of $A$ that $R_{A}^{\perp}=N_{A}$ and $R_{A}=P_{A}$.

Corollary 1 (To Lemma 1). For $k<n$, suppose there is no $d_{k+1}$ such that $A c_{k+1} \neq 0$. Then for any $x, y$ such that $y=A x$,

$$
A\left(A_{k} y-x\right)=0 .
$$

Proof. First, we prove that $A c_{i}, i \leqq k$, span $R_{A}$. If this is not true, then, for some $z \neq 0$ in $R_{A}, z^{\prime} A c_{i}=0, i \leqq k$, and (since $\left.R_{A}=P_{A}\right) A z \neq 0$; then, for $d_{k+1}=z$,

$$
A_{k} A d_{k+1}=\sum_{i=1}^{k} \frac{c_{i} c_{i}^{\prime}}{c_{i}^{\prime} A c_{i}} A d_{k+1}=0
$$


and

$$
c_{k+1}=d_{k+1}-A_{k} A d_{k+1}=d_{k+1}=z .
$$

Hence, we have found a $d_{k+1}$ such that

$$
A c_{k+1}=A z \neq 0,
$$

a contradiction to the main hypothesis. Thus, $A c_{i}, i \leqq k$, span $R_{A}$. Hence, for any $x$, $A x=\sum_{i=1}^{k} a_{i} A c_{i}$ so that for $y=A x$, we have

$$
\begin{aligned}
A\left(A_{k} y-x\right) & =A A_{k} A x-A x \\
& =A\left[\sum_{i=1}^{k} \frac{c_{i} c_{i}^{\prime}}{c_{i}^{\prime} A c_{i}} \sum_{i=1}^{k} a_{i} A c_{j}\right]-\sum_{j=1}^{k} a_{i} A c_{j}=0 .
\end{aligned}
$$

The algorithm is modified as follows. Suppose $f$ is given by (II.1), $A$ is a p.s.s.m., and there is no step $d_{k}$ such that in Step (1) of the algorithm, $a_{k} \neq 0$. Since $a_{k}=$ $s_{k}^{\prime} y_{k}=s_{k}^{\prime} A s_{k}, a_{k}>0$ if and only if $A s_{k} \neq 0$; hence, $A s_{k}=0, A$ is of rank $k-1$, and $A s_{i}, i \leqq k-1$, span $R_{A}$ (see above proof). We will show that for any $x, f$ is minimized by

$$
z=x-A_{k-1} g(x),
$$

where $g(x)=\operatorname{grad} f(x)=A x+b$. First, note that $\hat{x}$ minimizes $f$ if and only if $g(\hat{x})=0$. For any such $\hat{x}$, let

$$
\begin{aligned}
v & =A_{k-1} g(x)-(x-\hat{x})=A_{k-1}(g(x)-g(\hat{x}))-(x-\hat{x}) \\
& =A_{k-1} A(x-\hat{x})-(x-\hat{x}),
\end{aligned}
$$

where, by Corollary $1, A 0=0$, since there is no $d_{k}$ such that $A s_{k} \neq 0\left(a_{k} \neq 0\right)$. Then,

$$
z=x-A_{k-1} g(x)=\hat{x}-v
$$

and

$$
g(z)=A(\hat{x}-v)+b=A \hat{x}+b=g(\hat{x})=0 .
$$

Thus, $z$ minimizes $f$. In particular, $x_{k-1}-A_{k-1} g_{k-1}$ minimizes $f$.

In order to make use of Corollary 1 , we must be able to determine that there is no $d_{k}$ such that $a_{k} \neq 0$. This is easily accomplished by trying $N-(k-1)$ directions independent of $d_{1}, \cdots, d_{k-1}$, determining these directions by use of the GramSchmidt procedure given in Appendix I (by (2) of Lemma 1, we do not have to try $d_{1}, \cdots, d_{k-1}$ or any combination of these).

It is often desirable to find $A, A^{*}$, and the vector of least norm that minimizes $f$ (least squares minimum of $f$ ). Once a minimizing solution has been obtained, these are all easily found by straightforward application of the following results. Regarding notation used below, note that in the algorithm, $A s_{i}=y_{i}$ when $f$ is given by (II.1).

Lemma 4. (1) Let $s_{1}, \cdots, s_{k}$ be an $A$-orthogonal basis for $P_{A}$. Then $\hat{A}=A^{*}$, where

$$
\hat{A}=\sum_{i=1}^{k} \frac{s_{i} s_{i}^{\prime}}{s_{i}^{\prime} A s_{i}}
$$


(2) Let $z_{1}, \cdots, z_{k}$ be an $A^{*}$-orthogonal basis for $P_{A}^{*}$.Then,

$$
A=\sum_{i=1}^{k} \frac{z_{i} z_{i}^{\prime}}{z_{i}^{\prime} A^{*} z_{i}}
$$

Suppose that in Step (1) of the algorithm, there is no $d_{k}$ such that $a_{k} \neq 0$, and $f$ is given by (II.1). Then,

(3) $A=\sum_{i=1}^{k-1} A s_{i} s_{i}^{\prime} A / s_{i}^{\prime} A s_{i}$.

(4) Using $A$ from (3), an A-orthogonal basis $r_{i}, i \leqq k-1$, for $P_{A}$ can be generated from $A s_{i}, i \leqq k-1$, by the Gram-Schmidt orthogonalization procedure, viz. for - $i=1, \cdots, k-1$,

$$
\begin{aligned}
& M_{0}=0, \\
& r_{i}=A s_{i}-M_{i-1} A s_{i}, \\
& M_{i}=M_{i-1}+r_{i} r_{i}^{\prime} A / r_{i}^{\prime} A r_{i},
\end{aligned}
$$

then, by (1) of Lemma 4,

$$
A^{*}=\sum_{i=1}^{k-1} r_{i} r_{i}^{\prime} / r_{i}^{\prime} A r_{i}
$$

(5) Let $\hat{x}$ minimize $f$. Then, the least squares minimum of $f$ is $x^{*}$,

$$
x^{*}=A^{*} A \hat{x} .
$$

Proof. (1) For $x$ in $P_{A}\left(=R_{A}\right), x=\sum_{i-1}^{k} a_{i} A s_{i}$ and clearly $A \hat{A} x=x$. For $x$ in $R_{A}^{\perp}\left(=N_{A}\right), x^{\prime} s_{i}=0, i \leqq k$, and clearly $A x=0$.

(2) Follows from (1) of Lemma 4 and the fact that $\left(A^{*}\right)^{*}=A$.

(3) Since $A A^{*} A=A, s_{i}^{\prime} A A^{*} A s_{i}=s_{i}^{\prime} A s_{i}$, i.e., $A s_{i}, i \leqq k-1$, are $A^{*}$-orthogonal. Then $A s_{i}, i \leqq k-1$, are a basis for $P_{A}^{*}$ since they span $P_{A}^{*}$ (see proof of Corollary 1 ) and (3) follows from (2) of Lemma 4.

(4) Follows from (1) of Lemma 4, as stated.

(5) This is a property of the pseudo-inverse (see [7, Appendix C, 17.10]).

Executive Office of the President

Office of Emergency Preparedness

Systems Evaluation Division

Washington, D. C. 20504

1. R. Fletcher \& M. J. D. Powell, "A rapidly convergent descent method for minimization," Comput. J., v. 6, 1963, pp. 163-168. MR 27 \#2096.

2. B. A. Murtagh \& R. W. H. Sargent, A Constrained Minimization Method With Quadratic Convergence, presented at the I. M. A. Conference on Optimization, held at the University of Keele, 1968.

3. R. FletcheR, A New Approach to Variable Metric Algorithms, U.K.A.E.A. Report HL 69/4734.

4. M. J. D. Powell, "A survey of numerical methods for unconstrained optimization," SIAM Rev., v. 12, 1970, pp. 79-97. MR 41 \#2900.

5. H. H. ROSENBROCK, "An automatic method for finding the greatest or least value of a function," Comput.J., v. 3, 1960/61, pp. 175-184. MR 24 \#B2081.

6. H. W. SORENSON, "Comparison of some conjugate direction procedures for function minimization," J. Franklin Inst., v. 288, 1969, pp. 421-441. MR 40 \#233.

7. L. A. ZADEH \& C. A. Desoer, Linear System Theory, McGraw-Hill, New York, 1963.

8. H. J. Kelley \& G. E. Myers, Conjugate Direction Methods for Parameter Optimization, presented at the 18th Congress of the International Astronautical Federation, Belgrade, Yugoslavia, September 1967. 